\title{
A surprising link with unexplained infertility: a possible Covid-19 paradox?
}

\author{
Arianna Pacchiarotti ${ }^{1,2} \cdot$ Giacomo Frati $^{2} \cdot$ Pietro Saccucci $^{1}$
}

Received: 26 May 2020 / Accepted: 3 August 2020 / Published online: 26 August 2020

(C) Springer Science+Business Media, LLC, part of Springer Nature 2020

Unexplained infertility represents about $22-28$ percent of infertility causes [1].

The diagnosis is based on the exclusion of common causes using standard fertility investigation.

Over the years, the causes of unexplained infertility or subfertility have been identified in pre-existing associated pathologies, such as alterations of the immune system, thyroid dysfunctions, and coagulopathies.

Studies regarding the influence of psychological factors on the development of infertility highlighted that spontaneous pregnancies following adoption or the decision to remain childless are absolute exceptions. The link between stress and infertility in humans is still unclear. For many women, the effect of infertility and the subsequent medical therapy represents a considerable emotional stress [2].

Accordingly, the relationship between the environment and the reproductive capacity has been proved to have a direct correlation.

Previous work demonstrated that stress is associated with changes in eating habits and exercise, resulting in a complicated spectrum of reproductive disorders [3].

However, the potential impact of the interrelationship between stress and frequency of sexual intercourse on reproductive efficacy was not sufficiently described.

Arianna Pacchiarotti

arianna.pacchiarotti@aslroma1.it

Giacomo Frati

giacomo.frati@uniroma1.it

Pietro Saccucci

pietro.saccucci@aslroma1.it

1 IVF Unit, Ospedale San Filippo Neri, Via Giovanni Martinotti, 20 00135 Rome Italy

2 University of Rome Sapienza, Viale Regina Elena, Rome, Italy
The low frequency of sexual intercourse in the couple is often not mentioned during the anamnestic investigation, mostly because this issue is a source of frustration and shame.

Psychological stress, physical fatigue, lack of adequate time, and geographical distance represent the main determinants of low frequency of sexual intercourse. Therefore, there is a considerable proportion of couples with unexplained infertility that is treated with assisted reproduction techniques, who would have been able to naturally conceive a baby by simply increasing their sexual activity [4].

On March 10, Italy became the first democratic country since World War II to impose a national lockdown.

In few days, the outbreak spread from a northern crisis to a national one.

Restriction measures are imposed to stay home and seek permission for essential travel.

Many companies have started smart working for their employees.

At the same time, both the most accredited national and European scientific societies for reproductive medicine and the National Transplant Center, the institute that is responsible for the control of the Centres for ART (PMA) in Italy, have arranged to postpone the cycles of ARTs, waiting for the end of the coronavirus pandemic (Fig. 1).

In order to assess the potential impact of insufficient sexual activity on infertility, we studied whether recent COVID-19releated lockdown in Italy had any effect on conception ability of couples with unexplained infertility. We included in our study $N=50$ couples with unexplained infertility referring to our center at San Filippo Neri, Rome, Italy. In these couples, medically assisted procreation techniques were temporarily suspended because of the lockdown, as imposed by the Italian Institute of Health. The mean age of these patients was $38.8 \pm 1.8$ years old. AMH-assessed ovarian reserve was normal for the age. Among male partners, $66 \%(N=33)$ was normospermic, the remaining $34 \%(N=17)$ had moderate oligoasthenospermia. All the couples did not provide consistent information regarding their sexual activity. Ovulation 
Table 1 Baseline demographics of the patients

\begin{tabular}{llll}
\hline & $N=50$ & $N=7$ & $\begin{array}{l}P \text { value } \\
(P<0.05)\end{array}$ \\
\hline Mean age years $\pm \mathrm{sd}$ & $38.8 \pm 1.8$ & $38.5 \pm 0.8$ & $\mathrm{NS}$ \\
Normospermia \% $(N)$ & $66(33)$ & $71.4(N=5)$ & $\mathrm{NS}$ \\
Oligoasthenospermia \% $(N)$ & $34(17)$ & $28.6(N=2)$ & $\mathrm{NS}$ \\
Sexual activity per week $(N)$ & 0.5 & 10 & $<0.001$ \\
Caucasian ethnicity $(\%)$ & 100 & 100 & $\mathrm{NS}$ \\
BMI & $23 \pm 1.8$ & $24 \pm 1.9$ & $\mathrm{NS}$ \\
\hline
\end{tabular}

endocrine disorders or mild male factor were advocated as possible causes of infertility in these couples. BMI was $23 \pm$ 1.8

Interestingly, we found that 7 couples $(14 \%)$ conceived naturally after years (average $2 \pm 0.7$ years) of infertility. The mean age of these patients was $38.5 \pm 0.8$ years old, statistically not significant with mean age of the total group.

BMI was $24 \pm 1.9$ (Table 1).

All the seven couples were contacted by phone, and all confirmed a significant increase in sexual activity, from an average of $2 /$ month to two to three times/week $(p<0.001)$. They all stated that the longer time spent together at home contributed to the increase of their sexual activity.

It has to be noted that the spontaneous pregnancy rate is so significantly close to that in the literature of unexplained infertility $(14 \%$ vs $15 \%, p=0.9)$.

Therefore, lockdown and smart working during the pandemic have increased the frequency of sexual intercourse and could have unmasked the real cause of infertility or unexplained subfertility in these couples. It seems unlikely that a reduction of psychological stress would have contributed to the success to the natural conception of a baby in these seven couples, because of the extremely high stress levels felt in Italy during these months, in which more than 25,000 deaths due to COVID-19 were reported.

Our observation seems to suggest that insufficient sexual activity should always be ruled out in couples referring to reproductive centers for fertility issues. It is interesting to note that couples confined to home, naturally increase the amount of sexual intercourse. Clinicians probably should resist to the temptation to begin an active treatment and not immediately propose medical treatments in these couples. The real impact of insufficient sexual activity on unexplained infertility warrants further clarification in larger studies.

\section{References}

1. Kamath MS, Bhattacharya S. Demographics of infertility and management of unexplained infertility. Best Pract Res Clin Obstet Gynaecol. 2012;26(6):729-38.

2. Tewes H. Wischmann. Psychogenic infertility - myths and facts. J Assist Reprod Genet. 2003;20(12):485-94.

3. Negro-Vilar A. Stress and other environmental factors affecting fertility in men and women: overview. Environ Health Perspect Suppl. 1993;101(Suppl. 2):59-64.

4. Mol BW, Tjon-Kon-Fat R, Kamphuis E, van Wely M. Unexplained infertility: is it over-diagnosed and over-treated? Best Pract Res Clin Obstet Gynaecol. 2018;53:20-9 Review.

Publisher's note Springer Nature remains neutral with regard to jurisdictional claims in published maps and institutional affiliations. 